\title{
Renormalization of Self-consistent Approximation schemes at Finite Temperature II: Applications to the Sunset Diagram
}

\author{
Hendrik van Hees, Jörn Knoll \\ GSI Darmstadt, Planckstraße 1, D-64291 Darmstadt
}

(Dated: January 30, 2002)

\begin{abstract}
The theoretical concepts for the renormalization of self-consistent Dyson resummations, deviced in the first paper of this series, are applied to first example cases for the $\phi^{4}$-theory. Besides the tadpole (Hartree) approximation as a novel part the numerical solutions are presented which includes the sunset self-energy diagram into the self-consistent scheme based on the $\Phi$-derivable approximation or $2 \mathrm{PI}$ effective action concept.

PACS numbers: 11.10.-z, 11.10.Gh, 11.10.Wx
\end{abstract}

\section{INTRODUCTION}

In the first paper of this series [1] (in the following referred as I) we have derived the theoretical concepts for the renormalization of Dyson equation based resummation schemes at finite temperature. It could be shown that such truncated self-consistent approximations can be renormalized with counter term structures solely defined on the vacuum level of the considered approach, if two conditions are met: a) the underlying exact theory has to be renormalizable and b) the approximation scheme has to be based on Baym's $\Phi$-derivable concept [2, 3, 4], i.e., on a two-particle irreducible (2PI) effective action principle. Thus the self-consistent self-energies $\Sigma$ are generated from a truncated set of 2 PI closed diagrams (called $\Phi$ ) of the underlying full theory through

$$
\Sigma(p)=2 \mathrm{i} \frac{\delta \Phi[G]}{\delta G(p)},
$$

where $\Phi[G]$ is a functional of the self-consistent propagator $G(p)$ in momentum representation (as in I an asterix behind an equation number implies that the corresponding relations are only valid at the regularized level, while all other equations are valid also for the renormalized quantities). To repeat, the main issue is not to render any divergent loops finite, this has been pursued many times. The aim is to deploy the counter term structure such that it is entirely determined at the vacuum level of the self-consistent scheme. Besides the explicitely visible divergences this implies to resolve also the nested and overlapping vacuum divergences hidden in the self-consistent matter parts of the propagator.

In I we have given the proof that for the self-energy this can be done for any $\Phi$-derivable approximation, provided the underlying quantum field theory is renormalizable in the usual sense. Thereby the counter-term structure results from closed equations on the vacuum level, implicitly generating a particular though infinite subset of counter terms. The complexity of the ensuing equation is similar to that for the self-energy.

Thereby it is of particular importance that the entire counter term structure is consistently constructed solely and only within the effective action defined by the chosen approximation to $\Phi$. This implies that, e.g., the so obtained counter-term scheme and thus the behavior of the running coupling constant clearly deviates from the true one at orders beyond those included in $\Phi$. If these rules are not watched one may face uncompensated divergences as recently encountered in the detailed work of Braaten and Petitgirard [5]. They tackled the same problem, however using different techniques in terms of a restricted ansatz for the propagator. Uncompensated singularities 
arose from the fact that the used $\beta$-function was not the one pertaining to the self-consistent scheme but taken from the full $\beta$-function calculated up to the fifth loop order. Furthermore it is yet not transparent to us, how in their approach the hidden and nested vacuum subdivergences can be resolved such that one arrives at a counter term structure solely defined on the self-consistent vacuum level $(T=0)$. In our case the latter leads to a to be renormalized Bethe-Salpeter equation for the vacuum four-point function consistent with the chosen approximation level for the $\Phi$ functional.

In I we also have shown that the generating functional $\Gamma[\varphi, G]$ and thus the thermodynamic potential can be rendered finite with counter terms solely defined on the vacuum level of the self-consistent scheme. Again it is crucial to expand the functional around the solutions of the corresponding self-consistent approximation for the vacuum. It is not possible to render arbitrary parts of $\Gamma$ finite for themselves since it is important to use the stationarity of $\Gamma$ for the solution of the vacuum equations of motion. In particular terms linear in the matter parts of the propagator are singular and only drop out if the vacuum part is solved self-consistently!

For self-consistent Dyson resummation schemes it is well known [6], that they may violate symmetry properties such as crossing symmetry, masslessness of Nambu-Goldstone modes, conservation laws, etc. at a level beyond that of one-point functions, i.e., on the correlator level at orders beyond those included in $\Phi$. We devote a forthcoming paper [7] in this series to the issue of how to extend the scheme such that symmetry violations are indeed cured.

In this paper we give first numerical solutions for the leading and the next-to-leading order of the self-consistent Dyson equations for the $\phi^{4}$-theory, which is defined by the Lagrangian

$$
\mathscr{L}=\frac{1}{2}\left(\partial_{\mu} \phi\right)\left(\partial^{\mu} \phi\right)-\frac{m^{2}}{2} \phi^{2}-\frac{\lambda}{4 !} \phi^{4} .
$$

In the symmetric phase, $\langle\phi\rangle=0$, the first diagrams contributing to $\Phi$ and $\Sigma$ are

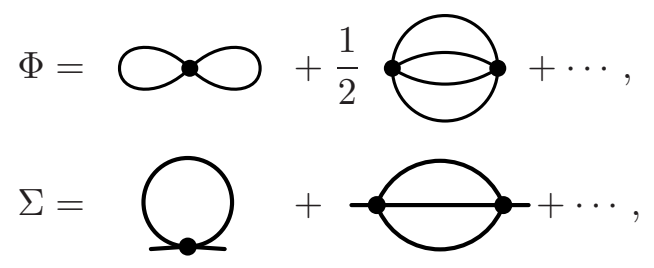

where all lines represent self-consistent propagators. The leading order diagram gives rise to the tadpole (Hartree) approximation for the self-energy, which, frequently considered in the literature (see for instance [6, 8]), leads to the standard gap equation. Here it is given as the most simple example for our general renormalization scheme. The next-to-leading order includes the sunset diagram for the self-energy. While in the tadpole case the self-energy is real and constant, with the sunset term the self-energy becomes momentum dependent and complex. Thus the particles acquire a finite spectral width due to collisions with the surrounding matter. With the sunset diagram one enters a new stage of sophistication, both, as far as the counter term structure is concerned, and with respect to the numerical solution of the resulting self-consistent equations.

First numerical investigations of the self-energy are presented which result from the two explicitly given diagrams in (4⿻ status given in [9] where we restricted ourselves to the computation of the imaginary parts of the self-energy to avoid problems with renormalization. The paper is organized as follows: In sect. 【I we briefly summarize the results of I as far as we need the formulae for the numerical calculations. In sect. III we solve the tadpole approximation (gap-equation) and in sect. IV the next-to-leading order approximation including the sunset diagram.

Throughout the paper we work in momentum-space representation within the real-time field theory formalism for the self-energies and propagators. Thus, if not stated otherwise, self-energies 
and Green's functions are contour matrix valued in the sense of the Schwinger-Keldysh real-time contour 10, 11, 12]. There vertices belonging to time arguments on the time and anti-time ordered branches are labeled by - and + superscripts, respectively. The integration sense is accounted for by assigning the values $\pm \mathrm{i} \lambda$ to the bare vertex depending on its placement on the $\{-\}$ or $\{+\}$ branches of the contour.

\section{THE RENORMALIZED EQUATIONS OF MOTION}

In I we have shown that arbitrary self-consistent approximations given by the $\Phi$-derivable concept (I) can be renormalized with counter terms defined on the vacuum level. Thereby the renormalized self-energy as well as the thermodynamical potential are finite and consistent to one another.

For the renormalization procedure the self-energy has to be split into three parts, namely the pure vacuum part (which is of divergence degree $\delta\left(\Sigma^{(\mathrm{vac})}\right)=2$ ), the part $\Sigma^{(0)}$ with divergence degree $\delta\left(\Sigma^{(0)}\right)=0$, containing explicit and hidden vacuum sub-divergences, and the rest $\Sigma^{(\mathrm{r})}$ with $\delta\left(\Sigma^{(\mathrm{r})}\right)=-2$, which contains only explicit vacuum sub-divergences with corresponding counterterms

$$
\Sigma=\Sigma^{(\mathrm{vac})}+\Sigma^{(0)}+\Sigma^{(\mathrm{r})} .
$$

The self-consistent Green's function $G$ follows from the Dyson equation in contour matrix form

$$
\Delta^{-1} G=1+\Sigma G \text {. }
$$

Here $\Delta$ is the free Green's function on the contour. In view of (5) the self-energy within the Green's function needs two subtractions to render its loops finite:

$$
\mathrm{i} G=\mathrm{i} G^{(\mathrm{vac})}+\underbrace{\mathrm{i} G^{(\mathrm{vac})} \Sigma^{(0, \mathrm{div})} G^{(\mathrm{vac})}+\mathrm{i} G^{(\mathrm{r})}}_{\mathrm{i} G^{(\text {matter })}}=-+-1 \mathrm{i} \Sigma^{(0)}-+\infty
$$

The subtraction only affects contour diagonal pieces of the propagator, since loops containing mixed contour vertices are finite for themselves. Thus

$$
\mathrm{i} G^{(\text {vac })}(p)=\left(\begin{array}{cc}
\mathrm{i} G^{--(\operatorname{vac})}(p) & 0 \\
0 & \mathrm{i} G^{++(\operatorname{vac})}(p)
\end{array}\right), \quad \mathrm{i} \Sigma^{(0 ; \operatorname{div})}(p)=\left(\begin{array}{cc}
\mathrm{i} \Sigma^{--(0)}(p) & 0 \\
0 & \mathrm{i} \Sigma^{++(0)}(p)
\end{array}\right),
$$

and likewise $\Sigma^{(\mathrm{vac})}$ are diagonal. While $G^{(\mathrm{vac})}$ and $\Sigma^{(\mathrm{vac})}$ are given by the (anti-)time ordered selfconsistent expressions given by the $\Phi$-derivable scheme on the vacuum level, $\Sigma^{(0)}$ accounts for the self-energy parts linear in $G^{\text {(matter) }}$. The three r.h.s. terms of (7) are of divergence degree $-2,-4$, and -6 , respectively. It is important to notice that only the full self-consistent propagator and selfenergy, $G$ and $\Sigma$, obey the equilibrium conditions (KMS) among their four contour components, cf. Eqs. (A.14) to (A.18) in I. The components of subtracted pieces like $G^{(\text {matter })}, \Sigma^{(0)}$ or $\Sigma^{(\mathrm{r})}$ obey no direct equilibrium relations as they result from differences of finite temperature with vacuum terms. Here we see an advantage in the real-time formalism which naturally permits this decomposition into vacuum and matter pieces of all dynamical quantities.

The contour diagonal parts of $\Sigma^{(0)}$ and $\Sigma^{(\mathrm{r})}$ contribute only at finite temperature. Due to Lorentz invariance the vacuum self-energy and the Green's function are functions of $s=p^{2}$. The vacuum self-energy is renormalized according to the rules of the on-shell renormalization scheme, i.e.,

$$
\Sigma^{(\mathrm{vac})}\left(s=m^{2}\right)=0, \quad \partial_{s} \Sigma^{(\mathrm{vac})}\left(s=m^{2}\right)=0,
$$


which defines $m$ to be the physical mass of the particles and normalizes the wave functions such that the residuum of the Green's function at $s=m^{2}$ is equal to 1 . As we shall see in the following the renormalization procedure used to calculate the vacuum parts, and subsequently needed for the temperature dependent pieces of the self-energy, allows to chose any renormalization scheme. Especially it is possible to use "mass independent" minimal subtraction schemes (MS [13] or $\overline{\mathrm{MS}}$ [14] of dimensional regularization) so that the renormalization program shown in I is applicable also for the massless case. For the renormalization of the self-energy and the 2 PI-functional $\Gamma[G]$ it is crucial to split all quantities into vacuum $(T=0)$ and finite temperature parts by the procedure given above.

As shown in I this procedure allows to define the generating functional in its dependence on the in-matter parts of the propagator with the self-consistent solution given by the stationary point. Simultaneously this leads to the renormalized thermodynamical potential. All divergences indeed compensate if and only if the vacuum level is solved and renormalized entirely within the same approximation level as used for the finite temperature parts. This fact together with the stationarity conditions of the generating functional at $T=0$ for the vacuum equations of motion avoids the singularity problems encountered in 5 .

In $\Sigma^{(0)}$ the in-matter part of the propagator is involved in logarithmically divergent loops which imply hidden divergences. As shown in I these can be resolved with the following result

$$
\Sigma^{(0)}(p)=\int \frac{\mathrm{d}^{4} l}{(2 \pi)^{4}}\left\{\left[\Gamma^{(4)}(l, p)-\Gamma^{(4, \mathrm{vac})}(l, 0)\right] G^{(\text {matter })}(l)+\Lambda(0, l) G^{(\mathrm{r})}(l)\right\} .
$$

Here in consistency with the Dyson equation the four-point function $\Gamma^{(4)}(p, q)$ is generated also from $\Phi$ through

$$
\Gamma^{(4)}(p, q)=2\left(\frac{\delta^{2} \Phi[G]}{\delta G(p) \delta G(q)}\right)_{T \rightarrow 0^{+}}^{(\mathrm{ren})} .
$$

The counter term $\Gamma^{(4, \mathrm{vac})}$ contains only the contour diagonal parts (all vertices placed on one side of the real-time contour) of $\Gamma^{(4)}$. This function is $2 \mathrm{PI}$ in the channel $p \rightarrow q$, i.e., one cannot disconnect the diagrams by cutting two lines and separating the lines carrying the momenta $p$ and $q$. It contains only vacuum pieces and can straight forwardly be renormalized with $\Gamma^{(4, \text { vac })}(0,0)= \pm \lambda / 2$ (denoted by the superscript (ren)). At the same time this four-point function defines the kernel for the vacuum $s$-channel Bethe-Salpeter equation defining the $2 \mathrm{PR}$ four-point function $\Lambda(p, q)$. In (10) we only need the half sided $\Lambda(0, p)$, which, as one of the crucial points of the BogoliubovParasiuk-Hepp-Zimmermann (BPHZ) renormalization procedure [15, 16], is given by the finite equation

$$
\Lambda(0, p)= \pm \frac{\lambda}{2}+\Gamma^{(4, \mathrm{vac})}(0, p)+\mathrm{i} \int \frac{\mathrm{d}^{4} l}{(2 \pi)^{4}} \Lambda(0, l)[G(l)]^{2}\left[\Gamma^{(4, \mathrm{vac})}(l, p)-\Gamma^{(4, \mathrm{vac})}(l, 0)\right],
$$

again involving only contour diagonal terms. Here we inserted the renormalization condition $\Lambda(0,0)= \pm \frac{\lambda}{2}$.

The expression (10) for $\Sigma^{(0)}$ void of the $\Lambda(0, l)$ part would correspond to a naive subtraction, which indeed is finite, however it implies $T$ dependent counter terms. As explained in I, only the entire expression (10) guarantees a counterterm structure solely defined on the self-consistent vacuum level.

\section{The perturbative view}

The above procedure defines the self-consistent renormalization scheme in terms of the corresponding selfconsistent vacuum propagator $G^{(\mathrm{vac})}$, the vacuum four-point function $\Lambda(0, l)$, and the self-consistent in-matter pieces 
of the propagator $G^{(\text {matter) }}$ or $G^{(\mathrm{r})}$. As an illustration we briefly quote the corresponding perturbative (1PI) view of the scheme, expanding the full self-energy in terms of the vacuum propagator in contour matrix notation satisfying the equilibrium relations (KMS) at the given temperature $T$

$$
\begin{aligned}
\mathrm{i} G^{(\operatorname{vac} ; T)}(p) & =\left(\begin{array}{cc}
\mathrm{i} G^{--(\mathrm{vac})}(p) & \begin{array}{c}
0 \\
0
\end{array} \\
\mathrm{i} G^{++(\operatorname{vac})}(p)
\end{array}\right)+\rho^{(\mathrm{vac})}\left(\left|p_{0}\right|, \vec{p}\right)\left(\begin{array}{cc}
n\left(p_{0}\right) & \Theta\left(-p_{0}\right)+n\left(p_{0}\right) \\
\Theta\left(p_{0}\right)+n\left(p_{0}\right) & n\left(p_{0}\right)
\end{array}\right) \\
& =
\end{aligned}
$$

with the vacuum spectral function and thermal Bose-Einstein weight defined by

$$
\begin{aligned}
\rho^{(\mathrm{vac})}(p) & =-2 \operatorname{Im} G_{R}^{(\mathrm{vac})}(p)=-2 \operatorname{sign}\left(p_{0}\right) \operatorname{Im} G^{--(\mathrm{vac})} \\
n\left(p_{0}\right) & =\frac{1}{\exp \left(\beta\left|p_{0}\right|\right)-1} .
\end{aligned}
$$

As discussed in I, loops involving the $\Theta$-functions or the thermal weights summarized by the dotted line are always finite.

For the self-consistent scheme of tadpole and sunset the following types of 1PI diagrams are resummed to the full self-consistent self-energy:

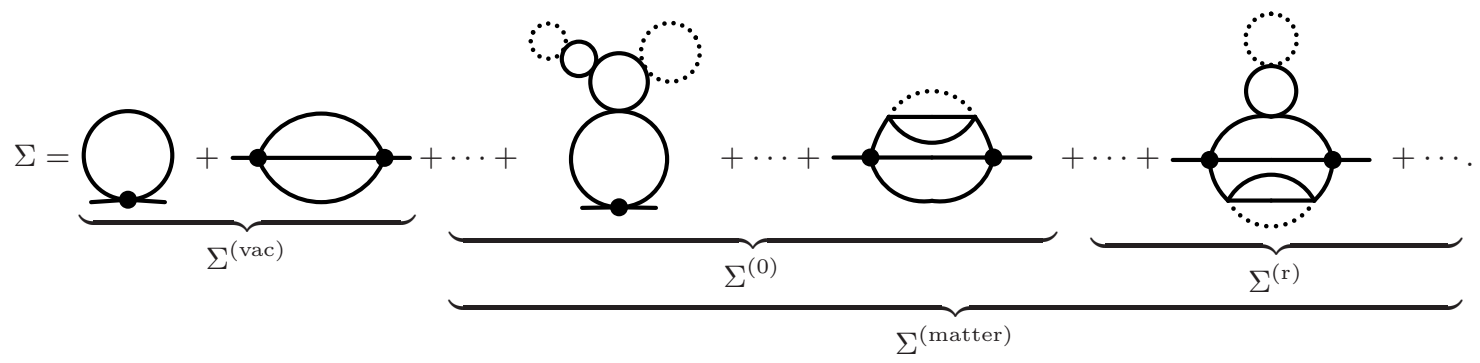

Here the diagrams for $\Sigma^{\text {(matter) }}$ are generated by iterative insertions of the diagrams of $\Sigma^{(\text {vac })}$, replacing one or more plain lines by dotted ones with the constraint that due to the self-consistence at the vacuum level all pure vacuum self-energy insertions are to be excluded. In $\Sigma^{\text {(matter) }}$ vacuum subdiagrams (plain lines) with 4 external lines are logarithmically divergent and need to be renormalized. Their sum defines the renormalized four point function $\Lambda$. If the latter include the external points (full dots) they contribute to $\Sigma^{(0)}$, defined in (月). All other terms, like the last term, contribute to $\Sigma^{(\mathrm{r})}$.

\section{THE TADPOLE APPROXIMATION}

The tadpole approximation is given by

$$
\begin{aligned}
\mathrm{i} \Phi[G] & =\bigcirc=\frac{\mathrm{i} \lambda}{8} \sum_{j= \pm} \sigma_{j j}\left[\int \frac{\mathrm{d}^{d} l}{(2 \pi)^{d}} G^{j j}(l)\right]^{2} \\
-\mathrm{i} \Sigma^{j j}=2 \mathrm{i} \frac{\delta \Phi[G]}{\delta G_{j j}} & =\bigcirc=\frac{\lambda}{2} \int \frac{\mathrm{d}^{d} l}{(2 \pi)^{d}} G^{j j}(l)=\Theta \sigma^{j j} . \quad j \in\{-,+\}
\end{aligned}
$$

for the un-renormalized $\Phi$-functional and self-energy. Here $\sigma=\operatorname{diag}(1,-1)$ accounts for the integration sense on the contour. The self-energy is diagonal in the contour indices. Since the tadpole self-energy is real and constant the vacuum part vanishes

$$
\Sigma^{(\mathrm{vac})}=0
$$

in view of the physical renormalization condition (9). To find the renormalized equations of motion at finite temperature we need the renormalized four-point function $\Lambda$ according to (10). Due to 
(11) we have a constant Bethe-Salpeter kernel

$$
\Gamma^{(4 ; \mathrm{vac})}=\frac{\lambda}{2}
$$

and thus $\Lambda$ is also constant and determined from its renormalization condition (12)

$$
\Lambda^{(\mathrm{vac})}=\frac{\lambda}{2}
$$

In the following it is sufficient to just consider the $\{--\}$-propagator. Writing $M^{2}=m^{2}+\Theta$ from (6) the self-consistent $\{--\}$-propagator is given by

$$
\begin{aligned}
& G^{--}(p)=\frac{1}{p^{2}-M^{2}+\mathrm{i} \eta}-2 \mathrm{i} \pi n_{T}\left(p_{0}\right) \delta_{\eta}\left(p^{2}-M^{2}\right) \\
& \text { with } \quad \delta_{\eta}(x)=\frac{1}{\pi} \operatorname{Im} \frac{1}{x-\mathrm{i} \eta}=\frac{1}{\pi} \frac{\eta}{x^{2}+\eta^{2}}
\end{aligned}
$$

and the Bose-Einstein function

$$
n_{T}\left(p_{0}\right)=\frac{1}{e^{\left|p_{0}\right| / T}-1} .
$$

Since the tadpole self-energy is constant it is identical with $\Sigma^{(0)}$ and due to (7) we thus have

$$
\begin{aligned}
G^{--(\mathrm{r})}(p) & =G^{--}(p)-G^{--(\mathrm{vac})}(p)-\Theta\left[G^{--(\operatorname{vac})}(p)\right]^{2} \\
& =\frac{\Theta^{2}}{\left(m^{2}-p^{2}-\mathrm{i} \eta\right)^{2}\left(M^{2}-p^{2}-\mathrm{i} \eta\right)}-2 \pi \mathrm{i} n_{T}\left(p_{0}\right) \delta_{\eta}\left(p^{2}-M^{2}\right) .
\end{aligned}
$$

This analytically given result explicitly illustrates that the subtracted part $G^{--(\mathrm{r})}$ of the propagator no longer obeys the finite temperature KMS and retarded relations (Eqs. (A.14)-(A.18) in I). The renormalized effective mass follows from (10) and (20) with standard integrals from vacuumperturbation theory (see for instance [17):

$$
M^{2}=m^{2}+\Theta=m^{2}+\frac{\lambda}{32 \pi^{2}}\left[M^{2} \ln \left(\frac{M^{2}}{m^{2}}\right)-M^{2}+m^{2}\right]+\frac{\lambda}{4 \pi^{2}} \int_{0}^{\infty} \mathrm{d} L L^{2} \frac{n_{T}\left(\sqrt{L^{2}+M^{2}}\right)}{\sqrt{L^{2}+M^{2}}} .
$$

The solution of this gap-equation for $m=0.14 \mathrm{GeV}$ and $\lambda=30$ is shown in Fig. 1 . The selfconsistent treatment reduces the mass gap relative to the perturbative result due to the larger mass $M$ entering the self-consistent loop.

The gap equation (24) becomes singular for $m \rightarrow 0$ due to the chosen on-shell renormalization condition (9). For the sake of completeness we give a brief summary about the treatment of the case of vanishing renormalized vacuum mass in the following.

For $m=0$ the renormalization description has to be changed to a so-called "mass independent" renormalization scheme. This concept is most easily established in the dimensional regularization procedure by the so called minimal subtraction (MS) or modified minimal subtraction $(\overline{\mathrm{MS}})$ schemes where for all renormalization parts, i.e., in our case of $\phi^{4}$-theory the proper $n$-point vertex functions with $n \leq 4$, only the singular terms in $\epsilon=(4-d) / 2$ are canceled.

In the present case of the tadpole approximation where the self-energies are momentumindependent this can be done analytically. Indeed for $m=0$ we have $\Theta=M^{2}$ and the divergent part of the diagram reads

$$
\Theta_{\infty}=\frac{\mathrm{i} \lambda \mu^{2 \epsilon}}{2} \int \frac{\mathrm{d}^{d} l}{(2 \pi)^{d}} \frac{1}{l^{2}-M^{2}+\mathrm{i} \eta}=-\frac{\lambda M^{2}}{32 \pi^{2}}\left[\frac{1}{\epsilon}+1-\gamma-\ln \left(\frac{M^{2}}{4 \pi \mu^{2}}\right)+O(\epsilon)\right]
$$




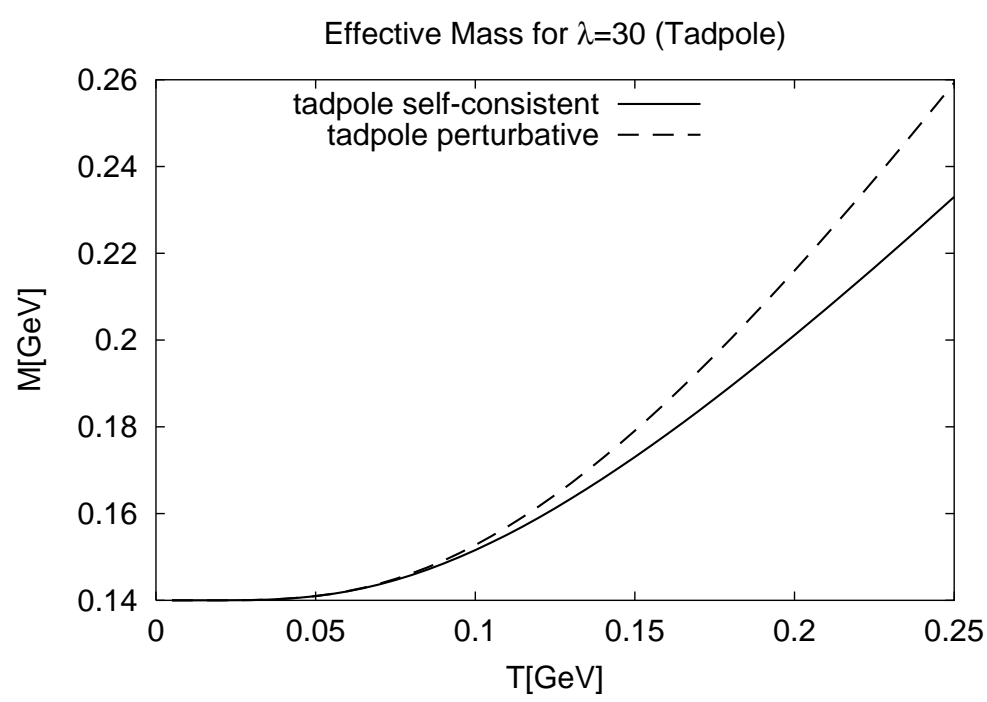

FIG. 1: The solution of the gap equation (24) compared to the naive, i.e., non-resummed one-loop perturbative solution. Both calculations are renormalized in the vacuum in the same way according to the on-shell conditions (9) with $m=0.14 \mathrm{GeV}$

in dimensional regularization. Since now the renormalized vacuum propagator is the free massless propagator, i.e.,

$$
G_{\mathrm{vac}}(l)=\frac{1}{l^{2}+\mathrm{i} \eta}
$$

an infrared regulating mass scale $\mu^{\prime}$ has to be introduced in order to calculate both, the mass and the four-point vertex

$$
\begin{aligned}
\Theta_{\text {vac }} & =\frac{\mathrm{i} \lambda \mu^{2 \epsilon}}{2} \int \frac{\mathrm{d}^{d} l}{(2 \pi)^{d}} \frac{1}{l^{2}-\mu^{\prime 2}+\mathrm{i} \eta}=-\frac{\lambda \mu^{\prime 2}}{32 \pi^{2}}\left[\frac{1}{\epsilon}+1-\gamma-\ln \left(\frac{\mu^{\prime 2}}{4 \pi \mu^{2}}\right)+O(\epsilon)\right], \\
\Gamma_{\text {vac }}^{(4)}(0) & =\frac{\mathrm{i} \lambda \mu^{2 \epsilon}}{2} \int \frac{\mathrm{d}^{d} l}{(2 \pi)^{d}} \frac{1}{\left(l^{2}-\mu^{\prime 2}+\mathrm{i} \eta\right)^{2}}=-\frac{\lambda}{32 \pi^{2}}\left[\frac{1}{\epsilon}-\gamma-\ln \left(\frac{\mu^{\prime 2}}{4 \pi \mu^{2}}\right)+O(\epsilon)\right]
\end{aligned}
$$

with the $\overline{\mathrm{MS}}$-counter terms

$$
\begin{aligned}
\delta m^{2} & =\lim _{\mu^{\prime} \rightarrow 0} \frac{\lambda \mu^{\prime 2}}{32 \pi^{2}}\left[\frac{1}{\epsilon}-\gamma\right]=0, \\
\delta \lambda & =\frac{\lambda}{32 \pi^{2}}\left[\frac{1}{\epsilon}-\gamma\right],
\end{aligned}
$$

which are IR-finite due to fact that in dimensional regularization the mass counter term in the $\overline{\mathrm{MS}}$ scheme is proportional to the infrared-regulator mass $\mu^{\prime 2}$. For $m=0$ the renormalized gap equation finally takes the form

$$
M^{2}=-\frac{\lambda M^{2}}{32 \pi^{2}}\left[1-\ln \left(\frac{M^{2}}{4 \pi \mu^{2}}\right)\right]+\frac{\lambda}{4 \pi^{2}} \int_{0}^{\infty} \mathrm{d} L L^{2} \frac{n_{T}\left(\sqrt{L^{2}+M^{2}}\right)}{\sqrt{L^{2}+M^{2}}}
$$

implying that perturbatively

$$
M_{\text {pert }}^{2}=\frac{\lambda}{4 \pi^{2}} \int_{0}^{\infty} \mathrm{d} L L n_{T}(L)=\frac{\lambda}{24} T^{2}
$$

which follows from (29) by letting $M \rightarrow 0$ on the r.h.s. 


\section{THE SUNSET APPROXIMATION}

In this section we calculate the self-consistent self-energy for the next-to-leading order approximation of the $\Phi$-functional numerically. First we have to solve the renormalized Dyson equation for the vacuum and the Bethe-Salpeter ladder equation for $\Lambda^{(\mathrm{vac})}$, which is needed as input for the temperature dependent calculation according to (10).

The $\Phi$-functional is given diagrammatically by

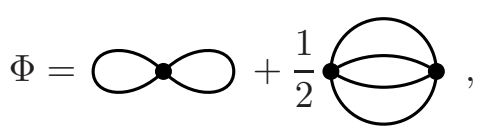

while the corresponding self-energy and BS-kernel become

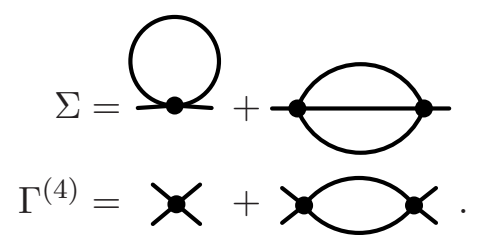

For the renormalization parts it is sufficient to restrict all considerations to one real-time contour branch. For definiteness we choose the time-ordered branch, i.e., in the following subsections all contour two-point functions denote $\{--\}$-quantities rather than matrices. The physical renormalization scheme (9) implies that the tadpole part of the self-energy is already subtracted such that in the vacuum case we only need to solve for the self-consistent sunset self-energy.

The general strategy will be to combine the BPHZ-renormalization scheme with dimensional regularization and use the spectral representation for the Green's functions (Lehmann representation)

$$
G^{(\mathrm{vac})}\left(p^{2}\right)=\int_{0}^{\infty} \frac{\mathrm{d}\left(m^{2}\right)}{\pi} \frac{\operatorname{Im} G^{(\mathrm{vac})}\left(m^{2}\right)}{m^{2}-p^{2}-\mathrm{i} \eta}
$$

All quantities like the renormalized vacuum self-energy $\Sigma^{(\mathrm{vac})}$, the BS-kernel $\Gamma^{(4)}$ and the four-point function $\Lambda$ are then to be expressed through the vacuum spectral function $\rho=-2 \operatorname{Im} G^{\text {(vac) }}$ and corresponding time-ordered kernel functions $K_{i}$. The latter express the analytic structure entirely in terms of free particle properties, however with varying masses. They can then be renormalized by dimensional regularization.

\section{A. The two-propagator loop}

A central quantity is the simple loop-function contained in (32 3 ) and (33*)

$$
L^{(\mathrm{reg})}\left(q^{2}\right)=\mathrm{i} \bigcirc=\mathrm{i} \int \frac{\mathrm{d}^{d} l}{(2 \pi)^{d}} G^{(\mathrm{vac})}\left[(l+q)^{2}\right] G^{(\mathrm{vac})}\left(l^{2}\right) .
$$

This four-point function is logarithmitically divergent and to be renormalized with the condition

$$
L^{(\text {ren })}(0)=0 .
$$

With help of the Lehmann representation (34) it can be expressed as

$$
L^{(\mathrm{ren})}\left(q^{2}\right)=\int_{0}^{\infty} \frac{\mathrm{d} m_{1}^{2}}{\pi} \int_{0}^{\infty} \frac{\mathrm{d} m_{2}^{2}}{\pi} K_{1}^{(\mathrm{ren})}\left(q^{2}, m_{1}^{2}, m_{2}^{2}\right) \operatorname{Im} G^{(\mathrm{vac})}\left(m_{1}^{2}\right) \operatorname{Im} G^{(\mathrm{vac})}\left(m_{2}^{2}\right)
$$


with the renormalized kernel defined through

$$
\begin{aligned}
& K_{1}^{(\mathrm{ren})}\left(q^{2}, m_{1}^{2}, m_{2}^{2}\right)=K_{1}^{(\mathrm{reg})}\left(q^{2}, m_{1}^{2}, m_{2}^{2}\right)-K_{1}^{(\mathrm{reg})}\left(0, m_{1}^{2}, m_{2}^{2}\right), \\
& K_{1}^{(\mathrm{reg})}\left(q^{2}, m_{1}^{2}, m_{2}^{2}\right)=\mathrm{i} \int \frac{\mathrm{d}^{d} l}{(2 \pi)^{d}} \frac{\mu^{2 \epsilon}}{\left(m_{1}^{2}-l^{2}-\mathrm{i} \eta\right)\left[m_{2}^{2}-(l+p)^{2}-\mathrm{i} \eta\right]} .
\end{aligned}
$$

Here the standard notation for dimensionally regularized quantities is used, where $d=4-2 \epsilon$ is the space-time dimension, and $\mu$ denotes the renormalization scale.

After a Feynman parameterization the integral (38) can be expressed in terms of standard formulae of dimensional regularization (see, e.g., [17]) which leads to the result

$$
\begin{aligned}
& K_{1}^{(\mathrm{reg})}\left(s, m_{1}^{2}, m_{2}^{2}\right)=\frac{1}{16 \pi^{2} s}\left\{-\left(\frac{1}{\epsilon}+2-\gamma\right) s+\right. \\
& +\lambda\left(s, m_{1}, m_{2}\right)\left[\operatorname{artanh}\left(\frac{s+m_{1}^{2}-m_{2}^{2}}{\lambda\left(s, m_{1}^{2}, m_{2}^{2}\right)}\right)+\operatorname{artanh}\left(\frac{s-m_{1}^{2}+m_{2}^{2}}{\lambda\left(s, m_{1}^{2}, m_{2}^{2}\right)}\right)\right]+ \\
& \left.+\left(m_{1}^{2}-m_{2}^{2}\right) \ln \left(\frac{m_{1}}{m_{2}}\right)+s \ln \left(\frac{m_{1} m_{2}}{4 \pi \mu^{2}}\right)\right\} \text {. }
\end{aligned}
$$

with the Källén function

$$
\lambda\left(s, m_{1}^{2}, m_{2}^{2}\right)=\sqrt{s^{2}+m_{1}^{4}+m_{2}^{4}-2 s m_{1}^{2}+-2 s m_{2}^{2}-2 m_{1}^{2} m_{2}^{2}},
$$

which is completely symmetric in its 3 arguments and the branch is determined by the $s+\mathrm{i} \eta$ prescription. Here and in the following we neglect contributions of order $O(\epsilon)$. For the renormalization of this kernel we also need its value at $s=0$ given by

$$
\begin{aligned}
K_{1}^{(\mathrm{reg})}\left(0, m_{1}, m_{2}\right)=\frac{1}{16\left(m_{1}^{2}-m_{2}^{2}\right) \pi^{2}}[ & -\frac{m_{1}^{2}-m_{2}^{2}}{\epsilon}-1+\gamma \\
& \left.+m_{1}^{2} \ln \left(\frac{m_{1}^{2}}{4 \pi \mu^{2}}\right)-m_{2}^{2} \ln \left(\frac{m_{2}^{2}}{4 \pi \mu^{2}}\right)\right] .
\end{aligned}
$$

According to 38 we thus find

$$
\begin{aligned}
K_{1}^{(\mathrm{ren})}\left(s, m_{1}, m_{2}\right)= & {\left.\left[K_{1}^{(\mathrm{reg})}(s)-K_{1}^{(\mathrm{reg})}(0)\right]\right|_{\epsilon \rightarrow 0} } \\
= & \frac{1}{16 \pi^{2} s}\left\{-s+\frac{\left(m_{1}^{2}-m_{2}^{2}\right)^{2}-s\left(m_{1}^{2}+m_{2}^{2}\right)}{m_{1}^{2}-m_{2}^{2}} \ln \left(\frac{m_{1}}{m_{2}}\right)\right. \\
& \left.+\lambda\left(s, m_{1}, m_{2}\right)\left[\operatorname{artanh}\left(\frac{s+m_{1}^{2}-m_{2}^{2}}{\lambda\left(s, m_{1}^{2}, m_{2}^{2}\right)}\right)+\operatorname{artanh}\left(\frac{s-m_{1}^{2}+m_{2}^{2}}{\lambda\left(s, m_{1}^{2}, m_{2}^{2}\right)}\right)\right]\right\} .
\end{aligned}
$$

The result for the loop $L(s)$ with $s=q^{2}$ is given in Fig. 2. The imaginary part is essentially determined by the two-body phase-space which opens at $s=4 m^{2}$. To discuss the threshold singularity of $L$ it is sufficient to study the perturbative result obtained from the pole term of $G^{\text {(vac) }}$ which is analytically given as

$$
\begin{aligned}
L^{(\text {pert })}\left(s, m^{2}\right) & =K_{1}^{(\mathrm{ren})}\left(s, m^{2}, m^{2}\right)=\frac{1}{8 \pi^{2}}\left[\sqrt{\frac{s-4 m^{2}}{s}} \operatorname{artanh}\left(\sqrt{\frac{s}{s-4 m^{2}}}\right)-1\right] \\
& =\frac{1}{8 \pi^{2}}\left[\sqrt{\frac{s-4 m^{2}}{s}}\left(\ln \frac{\sqrt{s}+\sqrt{s-4 m^{2}}}{2 m}-\frac{\mathrm{i} \pi}{2}\right)-1\right] .
\end{aligned}
$$



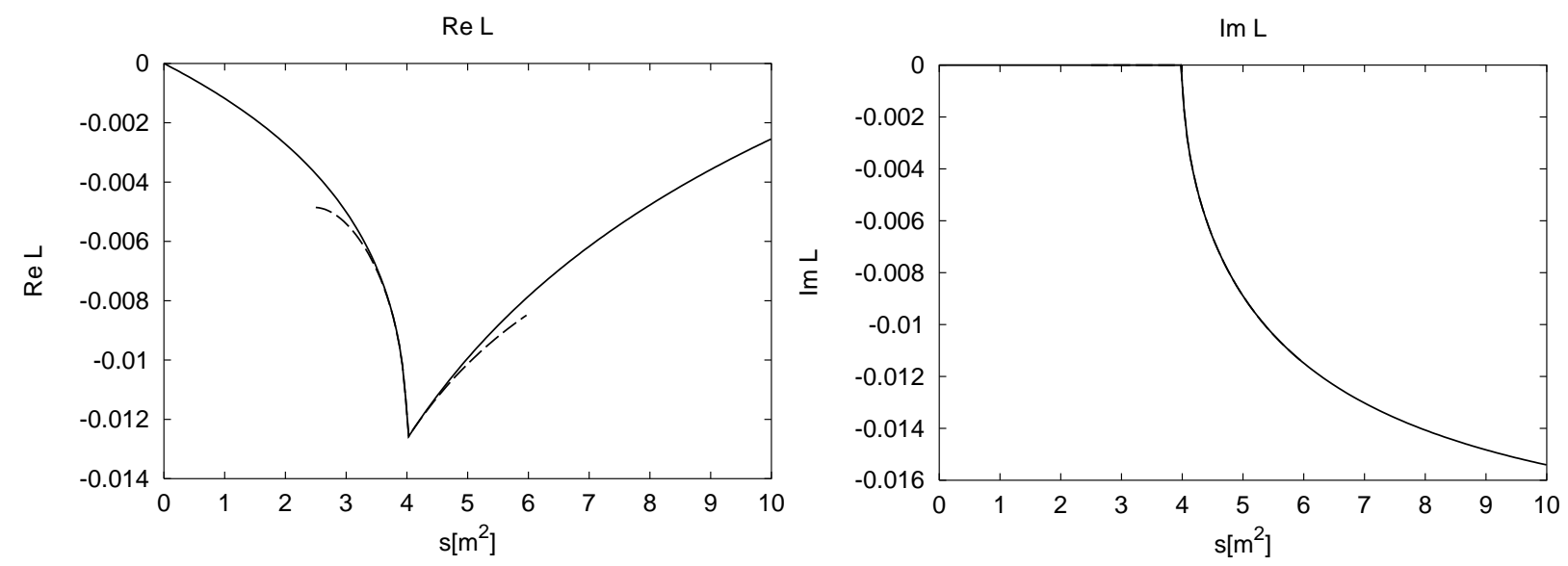

FIG. 2: Real (left) and imaginary part (right) of the loop function $L$. For the real part the dashed line shows the approximate behavior (44) around the threshold.

This function is analytic and real for real $s<0$. The analytic continuation to other values of $s$ is given by taking the principle branch of the square root and the artanh functions for $s+\mathrm{i} \eta$ with a small $\eta>0$. Close to threshold $s \approx 4 m^{2}$ one obtains the following approximate forms

$$
8 \pi^{2} L^{(\text {pert })}\left(s, m^{2}\right)=\left\{\begin{array}{l}
-\frac{\pi}{2} \sqrt{\frac{4 m^{2}-s}{s}}-\frac{4 m^{2}}{s}+O\left(\left(\frac{s-4 m^{2}}{s}\right)^{2}\right) \text { for } 0<4 m^{2}-s \ll s, \\
\frac{\mathrm{i} \pi}{2} \sqrt{\frac{s-4 m^{2}}{s}}-\frac{4 m^{2}}{s}+O\left(\left(\frac{s-4 m^{2}}{s}\right)^{2}\right) \text { for } 0<s-4 m^{2} \ll s .
\end{array}\right.
$$

Around the threshold the square root term causes the singular behavior changing from real to imaginary values, while the remaining log-term is entirely real. At large $s$ the real part of $L$ behaves logarithmically, whereas the imaginary part goes to a constant.

\section{B. The vacuum self-energy}

The un-renormalized expression of the two-loop self-energy reads

$$
\Sigma^{(\mathrm{vac})}\left(p^{2}\right)=-\frac{\lambda^{2}}{6} \int \frac{\mathrm{d}^{d} l_{1}}{(2 \pi)^{d}} \int \frac{\mathrm{d}^{d} l_{2}}{(2 \pi)^{d}} G^{(\mathrm{vac})}\left(l_{1}^{2}\right) G^{(\mathrm{vac})}\left[\left(l_{1}+l_{2}+p\right)^{2}\right] G^{(\mathrm{vac})}\left(l_{2}^{2}\right) .
$$

With help of (37) the sunset self-energy with all sub-divergences subtracted becomes

$$
\bar{\Sigma}^{(\mathrm{vac})}(p)=\frac{\mathrm{i} \lambda^{2}}{6} \int \frac{\mathrm{d}^{d} l_{2}}{(2 \pi)^{d}} L\left[\left(l_{2}+p\right)^{2}\right] G^{(\mathrm{vac})}\left(l_{2}^{2}\right) .
$$

Now we apply the spectral representation for (35) with one subtraction determined by (36)

$$
L\left(q^{2}\right)=\int_{4 m^{2}}^{\infty} \frac{\mathrm{d}\left(m_{3}\right)^{2}}{\pi} \operatorname{Im} L\left(m_{3}^{2}+\mathrm{i} \eta\right)\left(\frac{1}{m_{3}^{2}-q^{2}-\mathrm{i} \eta}-\frac{1}{m_{3}^{2}-\mathrm{i} \eta}\right) .
$$

Together with the Lehman representation of the propagator (34) this leads to the renormalized vacuum self-energy

$$
\Sigma^{(\mathrm{vac})}(s)=\int_{4 m^{2}} \frac{\mathrm{d}\left(m_{3}^{2}\right)}{\pi} \int_{9 m^{2}} \frac{\mathrm{d}\left(m_{4}^{2}\right)}{\pi} K_{2}^{(\mathrm{ren})}\left(s, m_{3}^{2}, m_{4}^{2}\right) \operatorname{Im} L^{(\mathrm{ren})}\left(m_{3}^{2}\right) \operatorname{Im} G^{(\mathrm{vac})}\left(m_{4}^{2}\right),
$$


where due to the renormalization conditions (9) the kernel $K_{2}^{(\mathrm{ren})}$ is given by

$$
\begin{aligned}
K_{2}^{(\mathrm{ren})}\left(s, m_{3}^{2}, m_{4}^{2}\right)= & K_{1}^{(\mathrm{reg})}\left(s, m_{3}^{2}, m_{4}^{2}\right)-K_{1}^{(\mathrm{reg})}\left(m^{2}, m_{3}^{2}, m_{4}^{2}\right)- \\
& -\left(s-m^{2}\right)\left[\partial_{s} K_{1}^{(\mathrm{reg})}\left(s, m_{3}^{2}, m_{4}^{2}\right)\right]_{s=m^{2}} .
\end{aligned}
$$

The cancellation of the contributions from the subtraction of the sub-divergences is due to a specialty of the sunset-diagram: Here all contracted diagrams are of tadpole structure and therefore independent of the external momentum $p$ and thus are completely canceled by the overall subtractions. The advantage to take them nonetheless into account is that at any stage of the calculation we use renormalized functions which can be calculated numerically without using any intermediate regularized functions.

From (39) we find the analytical expression for the kernel $K_{2}$ :

$$
\begin{aligned}
K_{2}^{(\mathrm{ren})}\left(s, m_{3}^{2}, m_{4}^{2}\right)= & \frac{1}{16 \pi^{2} m^{2} s}\left\{m^{2} \lambda\left(s, m_{3}, m_{4}\right) \times\left[\operatorname{artanh}\left(\frac{s+m_{3}^{2}-m_{4}^{2}}{\lambda\left(s, m_{3}, m_{4}\right)}\right)\right.\right. \\
& \left.+\operatorname{artanh}\left(\frac{s-m_{3}^{2}+m_{4}^{2}}{\lambda\left(s, m_{3}, m_{4}\right)}\right)\right] \\
& -s \lambda\left(m^{2}, m_{3}, m_{4}\right) \times\left[\operatorname{artanh}\left(\frac{m^{2}+m_{3}^{2}-m_{4}^{2}}{\lambda\left(m^{2}, m_{3}, m_{4}\right)}\right)\right. \\
& \left.\left.+\operatorname{artanh}\left(\frac{m^{2}-m_{3}^{2}+m_{4}^{2}}{\lambda\left(m^{2}, m_{3}, m_{4}\right)}\right)\right]\right\} \\
& +\frac{\left(s-m^{2}\right)\left[\left(m_{3}^{2}-m_{4}^{2}\right)^{2}-m^{2}\left(m_{3}^{2}+m_{4}^{2}\right)\right]}{16 \pi^{2} m^{4} \lambda\left(m^{2}, m_{3}, m_{4}\right)} \\
& \times\left[\operatorname{artanh}\left(\frac{m^{2}+m_{3}^{2}-m_{4}^{2}}{\lambda\left(m^{2}, m_{3}, m_{4}\right)}\right)+\operatorname{artanh}\left(\frac{m^{2}-m_{3}^{2}+m_{4}^{2}}{\lambda\left(m^{2}, m_{3}, m_{4}\right)}\right)\right] \\
& -\frac{s-m^{2}}{16 \pi^{2} m^{2}}+\frac{\left(m_{3}^{2}-m_{4}^{2}\right)\left(s-m^{2}\right)^{2}}{16 \pi^{2} m^{4} s} \ln \left(\frac{m_{3}}{m_{4}}\right) .
\end{aligned}
$$

The equations (37) and (48), supplemented by the Dyson equation

$$
G^{(\mathrm{vac})}(p)=\frac{1}{p^{2}-m^{2}-\Sigma(\mathrm{vac})\left(p^{2}\right)+\mathrm{i} \eta},
$$

form the closed set of renormalized equations of motion, which were solved with the analytically given Kernels $K_{1}^{(\mathrm{ren})}$ and $K_{2}^{(\mathrm{ren})}$ from (42) and (50). For the integrals (37) and (48) a simple adaptive Simpson integrator was used. We have chosen $m=m_{\pi}=140 \mathrm{MeV}$ for the mass and $\lambda=30$. As Fig. 3 shows for this coupling there is no visible difference between the perturbative and the selfconsistent result since the main contribution comes from the pole term of the propagator, while the continuous part, which starts at a threshold of $s=9 \mathrm{~m}^{2}$, is suppressed. Thus, due to our renormalization scheme, where $m$ is the physical mass parameter, the pole-term result essentially coincides with the perturbative one.

\section{The Bethe-Salpeter function}

The renormalized Bethe-Salpeter kernel $\Gamma^{(4, \text { ren })}$ defined in (11) reads

$$
\Gamma^{(4, \text { ren })}(l, p):=\Gamma^{(4, \text { ren })}\left[(l-p)^{2}\right]=\frac{\lambda}{2}+\lambda^{2} L^{(\text {ren })}\left[(l-p)^{2}\right] .
$$



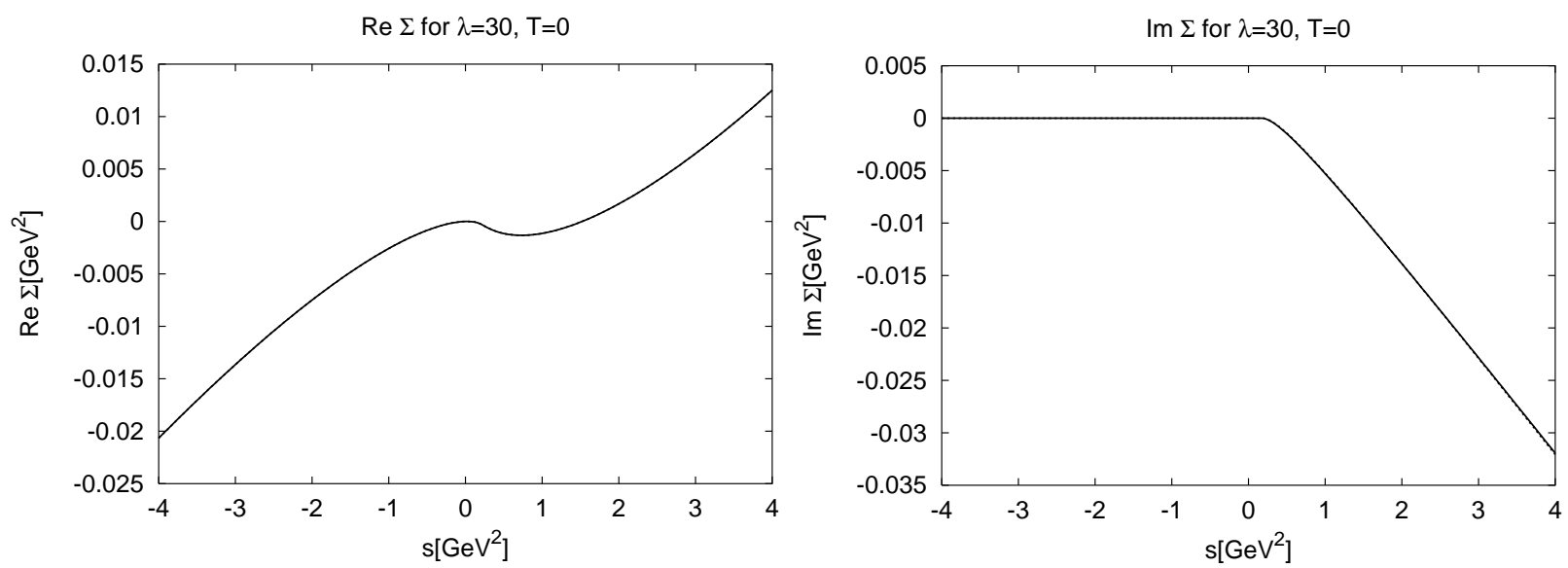

FIG. 3: Real (left) and imaginary part (right) of the sunset self- energy. The perturbative and the selfconsistent result lie on top of each other due to the large threshold at $s=9 \mathrm{~m}^{2}$.

Since as shown above the pole term essentially determines the loop-function $L$, we simplified the task to solve (10) by using the free propagator

$$
\Delta\left(p^{2}\right)=\frac{1}{p^{2}-m^{2}+\mathrm{i} \eta}
$$

instead of the self-consistent vacuum $G^{(\text {vac })}$.

Subtracted dispersion relations for $\Gamma^{(4, \text { ren })}$ and $\Lambda^{(\mathrm{ren})}$ and the renormalization conditions $\Gamma^{(4, \mathrm{ren})}(0,0)=\Lambda^{(\mathrm{ren})}(0,0)=\lambda / 2$ provide the renormalized integral equation

$$
\begin{aligned}
\Lambda^{(\mathrm{ren})}\left(0, p^{2}\right)= & \Gamma^{(4, \text { ren })}\left(p^{2}\right)+\mathrm{i} \int \frac{\mathrm{d}^{4} l}{(2 \pi)^{4}}\left[\Delta\left(l^{2}\right)\right]^{2} \times \\
& \times\left\{\int_{4 m^{2}}^{\infty} \frac{\mathrm{d}\left(m_{2}^{2}\right)}{\pi} \operatorname{Im} \Gamma^{(4, \text { ren })}\left(m_{2}^{2}\right) \frac{2 l p-p^{2}}{\left[m_{2}^{2}-(l-p)^{2}-\mathrm{i} \eta\right]\left(m_{2}^{2}-l^{2}-\mathrm{i} \eta\right)} \times\right. \\
& \left.\times\left[\int \frac{\mathrm{d}\left(m_{1}^{2}\right)}{\pi m_{1}^{2}} \frac{l^{2} \operatorname{Im} \Lambda^{(\mathrm{ren})}\left(0, m_{1}^{2}\right)}{m_{1}^{2}-l^{2}-\mathrm{i} \eta}+\frac{\lambda}{2}\right]\right\} .
\end{aligned}
$$

Again the $l$-integration can be performed with the help of standard perturbation theory integrals leading to the kernels

$$
\begin{aligned}
& K_{3}^{(\text {ren })}\left(p^{2}, m_{1}, m_{2}\right)=\mathrm{i} \int \frac{\mathrm{d}^{4} l}{(2 \pi)^{4}} \frac{1}{\left(m^{2}-l^{2}-\mathrm{i} \eta\right)^{2}} \frac{l^{2}\left(2 l p-p^{2}\right)}{\left(m_{1}^{2}-l^{2}-\mathrm{i} \eta\right)\left[m_{2}^{2}-(l-p)^{2}-\mathrm{i} \eta\right]}, \\
& K_{4}^{(\mathrm{ren})}\left(p^{2}, m_{2}\right)=\mathrm{i} \int \frac{\mathrm{d}^{4} l}{(2 \pi)^{4}} \frac{2 l p-p^{2}}{\left(m^{2}-l^{2}-\mathrm{i} \eta\right)^{2}\left[m_{2}^{2}-(l-p)^{2}-\mathrm{i} \eta\right]},
\end{aligned}
$$

which can be related to the previous kernel $K_{1}$ through the identity

$$
\left[\Delta\left(l^{2}\right)\right]^{2}=-\frac{1}{2 m} \partial_{m} \frac{1}{m^{2}-l^{2}-\mathrm{i} \eta} .
$$

We start with the calculation of $K_{4}$ defined in (55) and write it in the form

$$
K_{4}\left(s, m, m_{2}\right)=-\frac{\mathrm{i}}{2 m} \partial_{m} \int \frac{\mathrm{d}^{4} l}{(2 \pi)^{4}} \frac{1}{m^{2}-l^{2}-\mathrm{i} \eta}\left[\frac{1}{m_{2}^{2}-(l-p)^{2}-\mathrm{i} \eta}-\frac{1}{m_{2}^{2}-l^{2}-\mathrm{i} \eta}\right]
$$



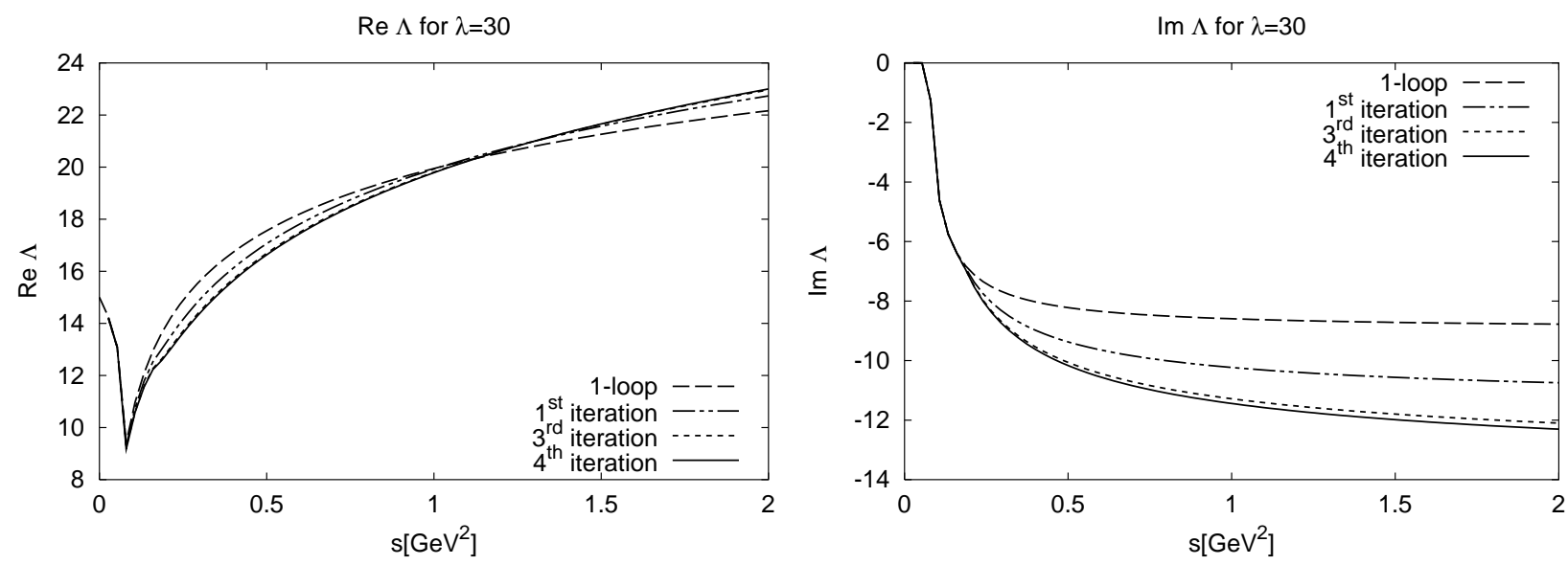

FIG. 4: Real (left) and imaginary part (right) of the $\Lambda$-function. The plot shows that after three iterations the solution is already stable.

which can be expressed in terms of $K_{1}^{(\text {ren })}$

$$
\begin{aligned}
K_{4}\left(s, m, m_{2}\right)= & -\frac{1}{2 m} \partial_{m} K_{1}\left(s, m, m_{2}\right)=-\frac{1}{16 \pi^{2} s}\left\{\frac{s}{m_{2}^{2}-m^{2}}+\frac{\left(m^{2}-m_{2}^{2}-s\right)}{\lambda\left(s, m, m_{2}\right)}\right. \\
& \times\left[\operatorname{artanh}\left(\frac{s+m_{1}^{2}-m_{2}^{2}}{\lambda\left(s, m_{1}, m_{2}\right)}\right)+\operatorname{artanh}\left(\frac{s-m_{1}^{2}+m_{2}^{2}}{\lambda\left(s, m_{1}, m_{2}\right)}\right)\right] \\
& \left.+\frac{\left(m^{2}-m_{2}^{2}\right)^{2}+2 m_{2}^{2} s}{\left(m^{2}-m_{2}^{2}\right)^{2}} \ln \left(\frac{m}{m_{2}}\right)\right\} .
\end{aligned}
$$

The integrand of $K_{3}$ cf. (55) can be rewritten as follows

$$
\begin{array}{r}
\frac{\mathrm{i}}{m^{2}-l^{2}-\mathrm{i} \eta} \frac{l^{2}}{m_{1}^{2}\left(m_{1}^{2}-l^{2}-\mathrm{i} \eta\right)} \frac{p^{2}-2 l p}{\left(m_{2}^{2}-l^{2}-\mathrm{i} \eta\right)\left[m_{2}^{2}-(l-p)^{2}-\mathrm{i} \eta\right]}= \\
=-\frac{\mathrm{i}}{2 m} \partial_{m} \frac{1}{m_{1}^{2}-m^{2}}\left(\frac{1}{m^{2}-l^{2}-\mathrm{i} \eta}-\frac{1}{m_{1}^{2}-l^{2}-\mathrm{i} \eta}\right)- \\
-\frac{1}{m_{1}^{2}\left(m^{2}-l^{2}-\mathrm{i} \eta\right)}\left[\frac{1}{m_{2}^{2}-(l-p)^{2}-\mathrm{i} \eta}-\frac{1}{m_{2}^{2}-l^{2}-\mathrm{i} \eta}\right] .
\end{array}
$$

The integration of (59) then leads to

$$
K_{3}\left(s, m, m_{1}, m_{2}\right)=\frac{K_{1}\left(s, m_{1}, m_{2}\right)-K_{1}\left(s, m, m_{2}\right)}{\left(m^{2}-m_{1}^{2}\right)^{2}}+\frac{m^{2}-2 m_{1}^{2}}{\left(m^{2}-m_{1}^{2}\right) m_{1}^{2}} K_{4}\left(s, m, m_{2}\right) .
$$

With the so defined kernels we can express (54) as follows:

$$
\begin{aligned}
\Lambda^{(\mathrm{ren})}\left(0, p^{2}\right)=\Gamma\left(p^{2}\right) & +\int_{4 m^{2}}^{\infty} \frac{\mathrm{d} m_{1}^{2}}{\pi m_{1}^{2}} \frac{\mathrm{d} m_{2}^{2}}{\pi} K_{3}\left(p^{2}, m_{1}, m_{2}\right) \operatorname{Im} \Lambda^{(\mathrm{ren})}\left(0, m_{1}^{2}\right) \operatorname{Im} \Gamma^{(4, \text { ren })}\left(m_{2}^{2}\right)+ \\
& +\frac{\lambda}{2} \int_{4 m^{2}}^{\infty} \frac{\mathrm{d} m_{2}^{2}}{\pi} K_{4}\left(p^{2}, m_{2}\right) \operatorname{Im} \Gamma^{(4, \text { ren })}\left(m_{2}^{2}\right) .
\end{aligned}
$$

Fig. As shows the solution of this renormalized equation of motion in comparison with the one-loop approximation $\Gamma^{(4, \text { ren })}$. As the figure shows, the solution is stable after three iterations. 


\section{The temperature dependent parts}

To calculate the temperature dependent part of the self-energy we use the analytical properties of the two-point functions and the fact that the retarded propagator fulfills the simple Dyson equation

$$
G_{R}(p)=\frac{1}{p^{2}-m^{2}-\Sigma_{R}(p)},
$$

where the retarded self-energy can be obtained from the $\{--\}$-matrix element via the equilibrium expression

$$
\Sigma_{R}(p)=\operatorname{Re} \Sigma^{--}(p)+\mathrm{i} \tanh \left(\frac{p_{0}}{2 T}\right) \operatorname{Im} \Sigma^{--}(p) .
$$

As explained in I both, $G^{(\text {vac })}$ and $\Sigma^{(0)}$, are diagonal matrices while the corresponding off-diagonal parts are contained in $G^{(\mathrm{r})}$, because they are of a lower degree of divergence due to explicit $\Theta+n$ factors.

Due to its topology the sunset-diagram has no further vertices besides its two external points. Therefore the 2PI vacuum four-point function $\Gamma^{(4, v a c)}$ is effectively only a two-point function and thus we need only the $\{--\}$-components of the various Green's functions in order to calculate $\Sigma^{--}$. This implies

$$
G^{--(\text {matter })}=G^{--}-G^{--(\mathrm{vac})}, \quad G^{--(\mathrm{r})}=G^{--(\text {matter })}-\left[G^{--(\mathrm{vac})}\right]^{2} \Sigma^{--(0)} .
$$

With help of the renormalized vacuum parts we obtain for the self-energy

$$
\begin{aligned}
\Sigma^{--}(p)= & \Sigma^{--(\mathrm{vac})}(p)+ \\
& +\mathrm{i} \int \frac{\mathrm{d}^{4} l}{(2 \pi)^{4}}\left\{\Gamma^{(4, \mathrm{vac})}\left[(l+p)^{2}\right]-\Gamma^{(4, \mathrm{vac})}\left(l^{2}\right)\right\} G^{--(\text {matter })}(l)+ \\
& +\mathrm{i} \int \frac{\mathrm{d}^{4} l}{(2 \pi)^{4}} \Lambda^{(\mathrm{ren})}\left(0, l^{2}\right) G^{--(\mathrm{r})}(l)- \\
& -\frac{\lambda^{2}}{2} \int \frac{\mathrm{d}^{4} l_{1}}{(2 \pi)^{4}} \int \frac{\mathrm{d}^{4} l_{2}}{(2 \pi)^{4}} G^{--(\text {matter })}\left(l_{1}\right) G^{--(\text {matter })}\left(l_{1}+l_{2}\right) G^{--(\mathrm{vac})}\left(l_{2}+p\right)- \\
& -\frac{\lambda^{2}}{6} \int \frac{\mathrm{d}^{4} l_{1}}{(2 \pi)^{4}} \int \frac{\mathrm{d}^{4} l_{2}}{(2 \pi)^{4}} G^{--(\text {matter })}\left(l_{1}\right) G^{--(\text {matter })}\left(l_{1}+l_{2}\right) G^{--(\text {matter })}\left(l_{2}+p\right) .
\end{aligned}
$$

Note that all vacuum quantities entering here are to be taken in their renormalized version and that the remaining integrals are all finite due to power counting.

\section{E. Results}

The above finite integrals are to be evaluated numerically. While due to Lorentz invariance the vacuum loops involve just two-dimensional integrations which can numerically be integrated by standard methods, for the in-matter loops only rotational symmetry in three momentum space can be exploited. For each loop diagram this leads to three dimensional integrals for each external momentum $p_{0},|\vec{p}|$. We solve these integrals on an equal distant $N \times N$ lattice in these coordinates. Naively the computing effort would then scale like $N^{5}$. However, we succeeded to develop an improved algorithm for the loop integrations, where the computing time scales with $N^{4}$ (essentially a gain of more than two orders of magnitude). The lattice implies an infrared cut-off which 
Pert. $\operatorname{Re} \Sigma$ for $\mathrm{T}=250 \mathrm{MeV}, \lambda=30$

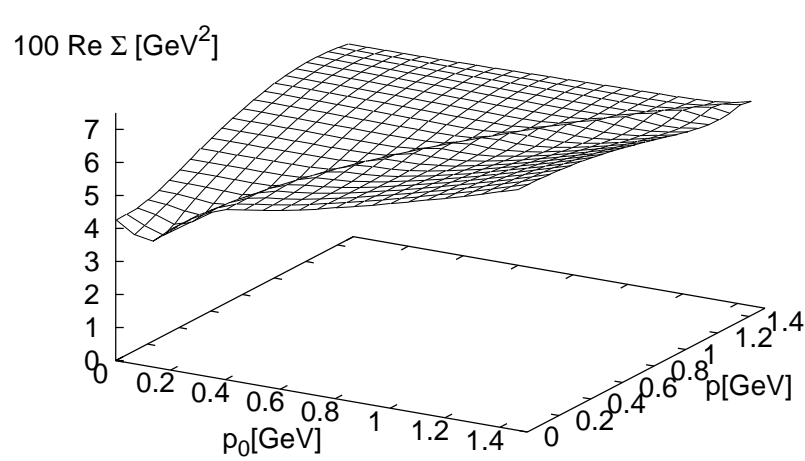

$\operatorname{Re} \Sigma$ for $\mathrm{T}=250 \mathrm{MeV}, \lambda=30$

$100 \operatorname{Re} \Sigma\left[\mathrm{GeV}^{2}\right]$

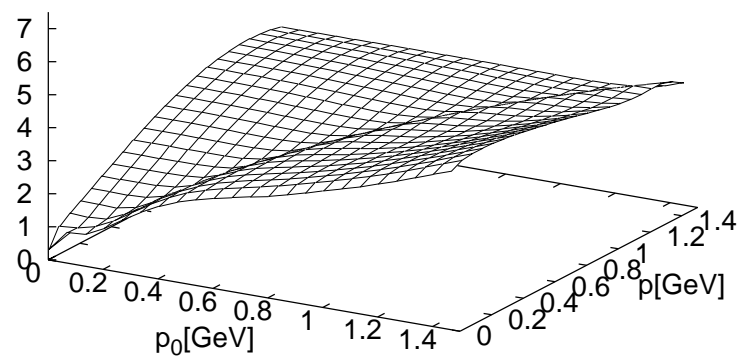

Pert. $\operatorname{Im} \Sigma$ for $\mathrm{T}=250 \mathrm{MeV}, \lambda=30$

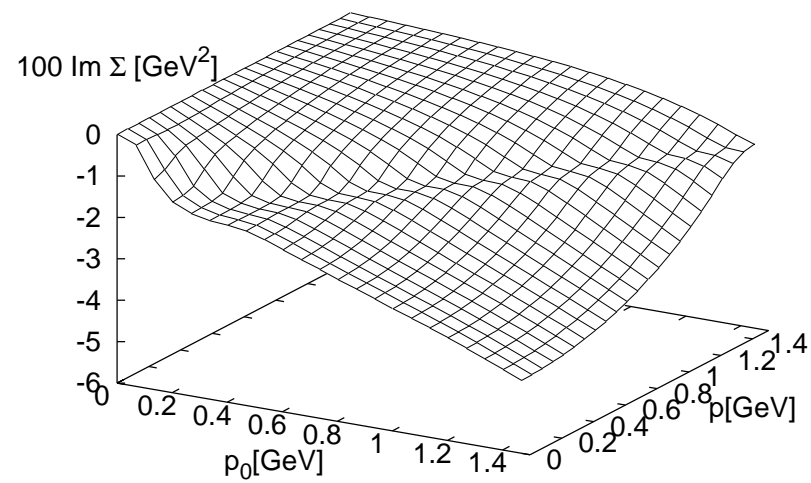

$\operatorname{Im} \Sigma$ for $\mathrm{T}=250 \mathrm{MeV}, \lambda=30$

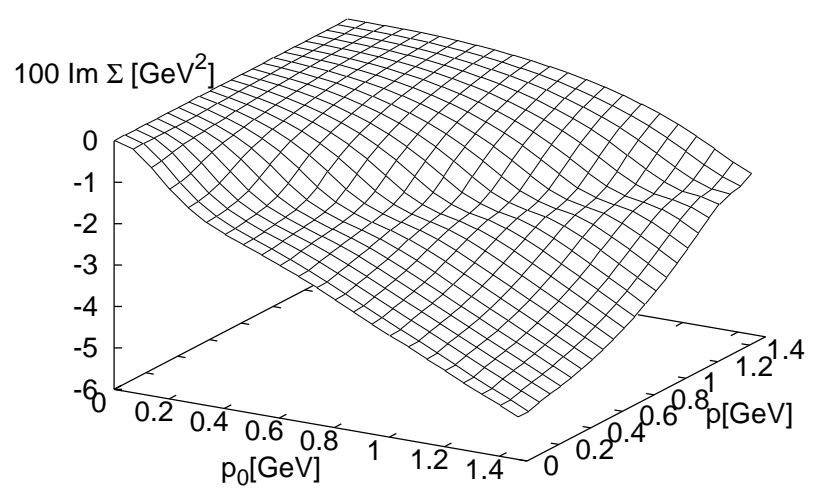

FIG. 5: Real (left) and imaginary part (right) of the perturbative (top) and the self-consistent self-energy for $\lambda=30, m=140 \mathrm{MeV}$ and $T=250 \mathrm{MeV}$. Note that the self-energies are multiplied with an factor $100 \mathrm{in}$ these plots!

requires particular care for the treatment of the sharp structures of the vacuum propagator around its on-shell pole. The method at hand was to tabulate the lattice cell integrated values of the propagator and its moment in $p_{0}$ given by the analytic result from a linear interpolation in $p_{0}^{2}$ of the inverse propagator at fixed $|\vec{p}|$. The remaining self-energy factors of the integrands are approximated to linear order in $p_{0}^{2}$. The interpolation procedure is adapted to the fact that due to the quadratic pole of the $\left(G^{--(\mathrm{vac})}\right)^{2}$ term one is sensitive to the $p_{0}$ derivative (residue) of the remaining integrand. Both, the infrared and the UV cut-offs of the lattice have been controlled by varying the lattice spacing and the overall size of the lattice. The final results were achieved with $\Delta p_{0}=\Delta|\vec{p}|=6.67 \mathrm{MeV}$ with $N=300$, i.e., a UV-cutoff at $2 \mathrm{GeV}$.

Perturbative results are obtained through Eq. (65) approximating $G^{\text {(matter) }}$ by the KMStemperature part of the free propagator $G^{(\mathrm{vac} ; T)}$ (last term in Eq. (13); note that here $G^{(\mathrm{r})}=$ $G^{\text {(matter) }}$ since $\left.\Sigma^{(\mathrm{vac})}=0\right)$. The self-consistent solutions are then obtained iteratively through the set of Eqs. (62) to (65). The results for both, the perturbative and the self-consistent case, are shown in Fig. 5 in a 3 -dimensional plot over the $\left(p_{0},|\vec{p}|\right)$-plane illustrating that the entire calculations are performed with the full dependence on energy and momentum. Details can be extracted from the cuts shown for a set of selected momenta in Fig. 6. The main qualitative results are similar for both the perturbative and the self-consistent calculation: In the vacuum and self-consistent pure tadpole case the self-energy shows a threshold cut resulting from the decay into three particles, i.e., $p_{0}^{2}-\vec{p}^{2} \geq 9 M^{2}$. Adding the sunset self-energy leads to a spectral width which 
Pert. Re $\Sigma$ for $\lambda=30, \mathrm{~T}=250 \mathrm{MeV}$

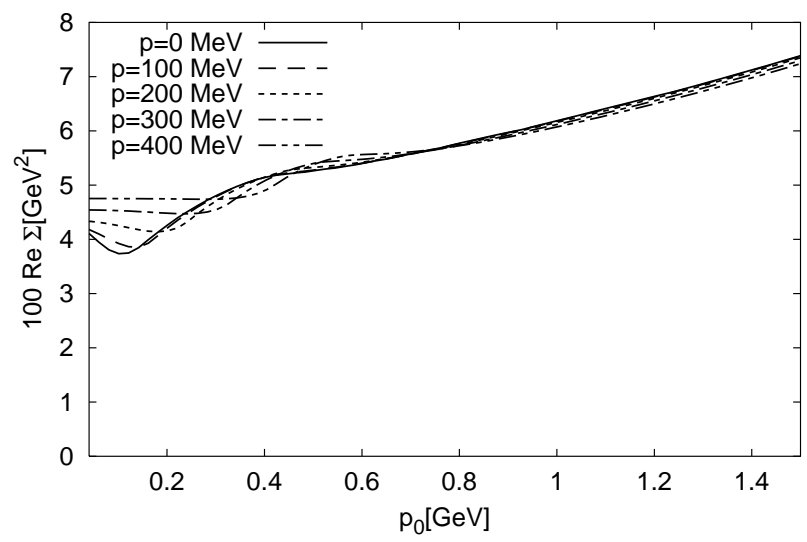

$\operatorname{Re} \Sigma$ for $\lambda=30, T=250 \mathrm{MeV}$

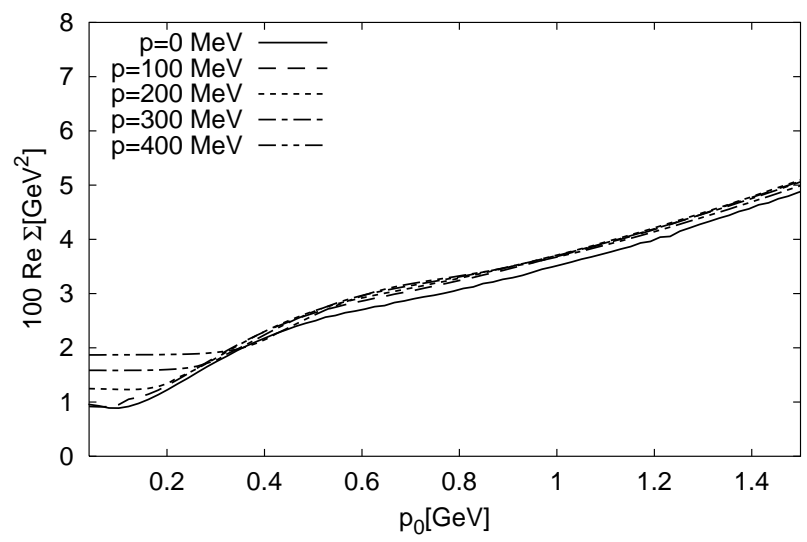

Pert. $\operatorname{Im} \Sigma$ for $\lambda=30, \mathrm{~T}=250 \mathrm{MeV}$

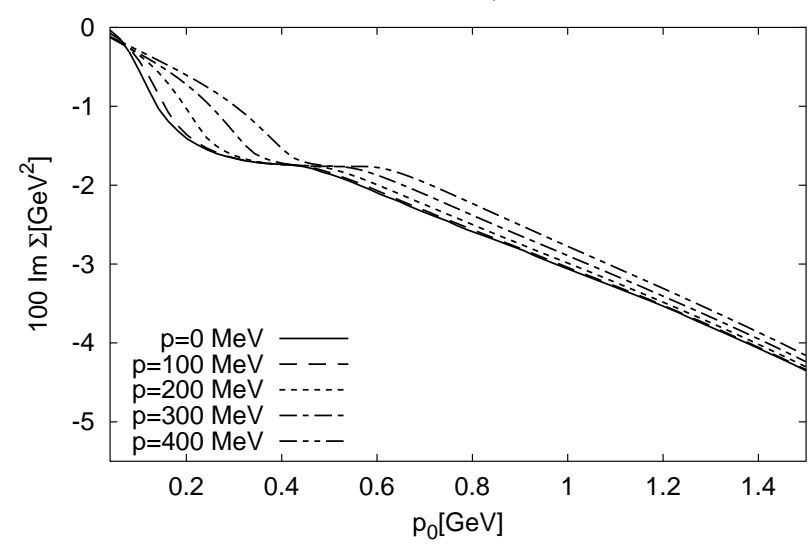

$\operatorname{Im} \Sigma$ for $\lambda=30, \mathrm{~T}=250 \mathrm{MeV}$

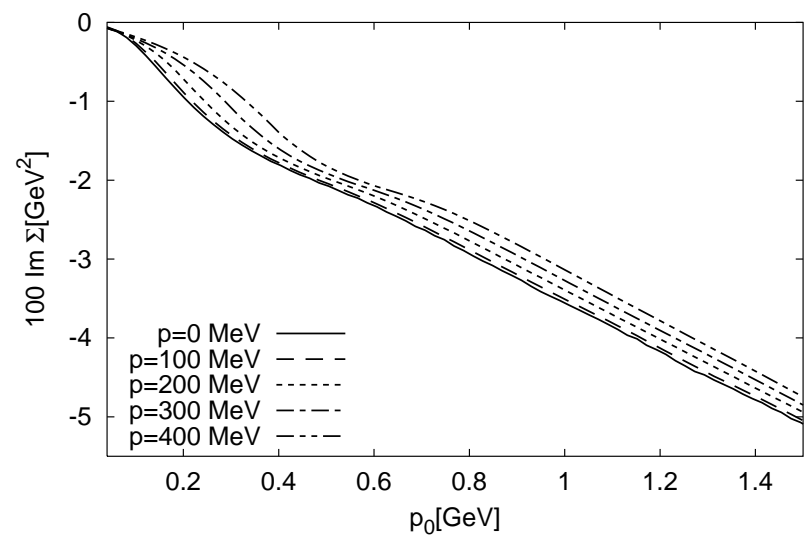

FIG. 6: Real (left) and imaginary part (right) of the perturbative (top) and the self-consistent self-energy for $\lambda=30, m=140 \mathrm{MeV}$ and $T=250 \mathrm{MeV}$ as a function of $p_{0}$ for various 3 -momenta. Note that the self-energies are multiplied by a factor of 100 in these plots!

dissolved this threshold such that the self-energy shows spectral strength (imaginary parts) at all energies. While the growing high-energy tail is related to the decay of virtual bosons into three particles, at finite temperature, as a new component, a low-energy plateau in $\operatorname{Im} \Sigma^{\mathrm{R}}$ emerges from in-medium scattering processes.

Various balancing effects are encountered for the self-consistent case: For sufficiently large couplings and/or temperatures the self-consistent treatment shows quantitative effects on the width. The finite spectral width itself leads to a further broadening of the width and a smoothing of the structures as a function of energy. This is however counter balanced by the behavior of the real part of the self-energy, which, as discussed below, essentially shifts the in-medium mass upwards. This reduces the available phase space for real processes. With increasing coupling strength $\lambda$ a nearly linear behavior of $\operatorname{Im} \Sigma^{\mathrm{R}}$ with $p_{0}$ results implying a nearly constant damping width given by $-\operatorname{Im} \Sigma^{\mathrm{R}} / p_{0}$.

The overall normalization of the real part of $\Sigma^{\mathrm{R}}$ is determined by the renormalization procedure. In this case there are three counter balancing effects. First the tadpole loop shifts the mass to higher values. As the tadpole is less effective for higher masses this effect weakens itself in the self-consistent tadpole treatment, c.f. Figs. 1 or 0 . However, since the sunset part adds spectral width, it indirectly contributes to the tadpole loop. Since spectral strength at the lower mass side 

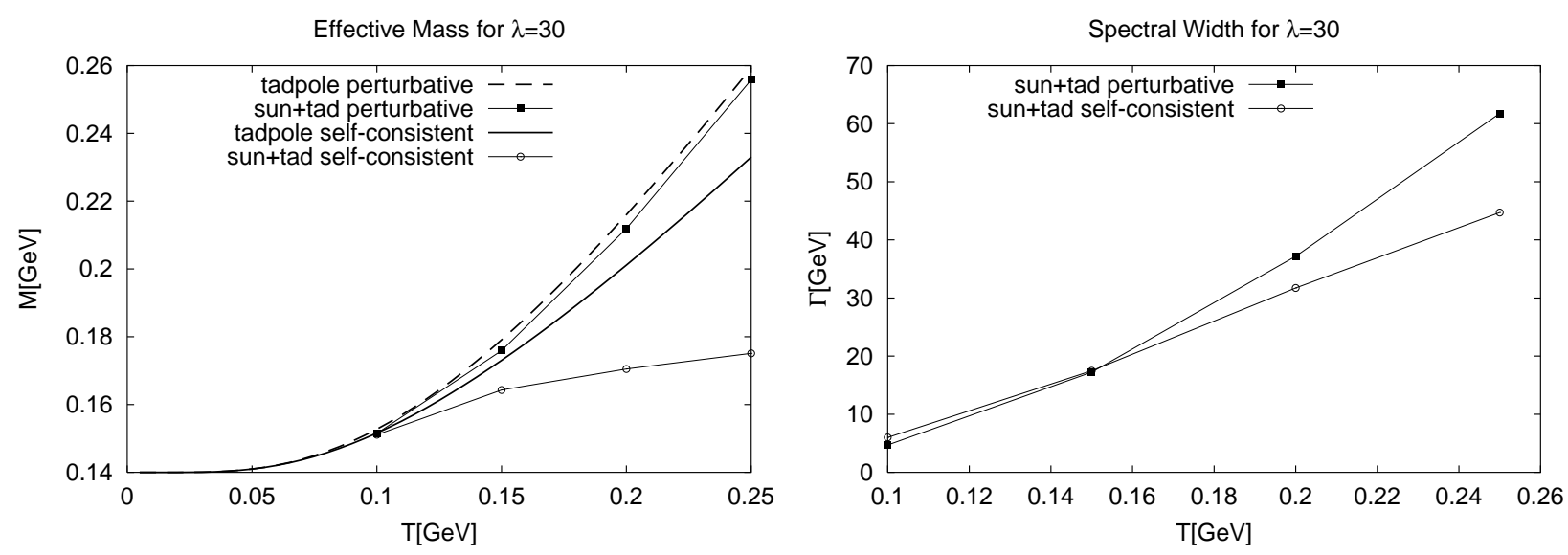

FIG. 7: The in-medium effective masses $M$ (left) and spectral widths $\Gamma$ (right) of the particles for the various approximations described in the text as a function of the system's temperature $T$.

carries higher statistical weights, the tadpole loop in turn leads to a further increase of the mass shift, c.f. the perturbative calculations of sunset \& tadpole in Fig. 7 .

The direct contributions of the sunset terms to the real part of the self-energy become relevant at higher couplings and temperatures. Then the self-consistency leads to significant effects which contributes to a net downshift of the real part of the self energy or in-medium mass $M$. The latter effect finally overrules the tadpole shift and indeed leads to an overall negative mass shift compared to the (tadpole dominated) perturbative result. These effects are illustrated in Fig. 7 where the in-medium effective mass $M$ and width $\Gamma$ of the corresponding "quasi-particles" are plotted against the temperature. Thereby $M$ and $\Gamma$ are defined as the quasi-particle energy $M=p_{0}$ at vanishing real part of the dispersion relation $\left[p_{0}^{2}-m^{2}-\operatorname{Re} \Sigma\left(p_{0}, \vec{p}\right)\right]_{p=(M, \overrightarrow{0})}=0$ for $\vec{p}=0$, and through $\Gamma=-\operatorname{Im} \Sigma(p) /\left.p_{0}\right|_{p=(M, \overrightarrow{0})}$, respectively.

\section{CONCLUSIONS AND OUTLOOK}

We have shown that it is possible to use the renormalization scheme, proposed in [1], for numerical investigations of the self-consistent approximations for the self-energy derived from the truncated effective action formalism on the $2 \mathrm{PI}$ level. Thereby it is very important to isolate the divergent vacuum parts consistently, in particular the implicit or hidden ones, from all convergent and in particular temperature or more generally matter-dependent parts. This could be provided by the ansatz given in I for both, the propagator and the self-energy. The renormalized vacuum pieces are obtained using the Lehmann representation for all two-point quantities. The resulting integration kernels can then be renormalized by standard technics. The procedure solely rests on Weinberg's power counting theorem, i.e., on an analysis of the asymptotic behavior of the propagators.

In this way both, the renormalized vacuum self-energy and four-point functions can directly be obtained from finite equations. For the finite temperature parts it is important that they have to be completely excluded from the counter term structures. This is achieved by the technique developed in I. Exploratory calculations are shown for the symmetric Wigner-Weyl phase of the $\lambda \phi^{4}$ model for the self-consistent treatment of both the tadpole and the sunset diagram at finite temperature.

The results promise that the method, which is conserving [2, 12] and thermodynamically consistent, can also be applied for the genuine non-equilibrium case, i.e., in quantum transport [18] 
or for the solution of the renormalized Kadanoff-Baym equations. Numerical studies for nonequilibrium cases (Gaussian initial conditions, spatially homogeneous systems) were already performed in [19, 20, 21]. These investigations were undertaken in $1+1$ dimensions. Our renormalization scheme should also be applicable for the non-equilibrium case in $1+3$ dimensions and implementable in numerical codes.

The investigation of the symmetry properties of $\Phi$-derivable approximations is the subject of a forthcoming publication [0]. It is known that in general the symmetries of the classical action which lead to Ward-Takahashi identities for the proper vertex-functions are violated for the self-consistent Dyson resummation for the functions beyond the one-point level, i.e., on the correlator level. The reason is that, although the functional $\Gamma$ can be expanded with respect to expansion parameters like the coupling or $\hbar$ (loop expansion) or large- $N$ expansions for $\mathrm{O}(N)$ type models, the solution of the self-consistent equations of motion contains partial contributions to any order of the expansion parameter. This resummation is of course incomplete and violates even crossing symmetry for the vertices involved in the renormalization procedure. This causes problems concerning the Nambu-Goldstone modes [6] in the broken symmetry case or concerning local symmetries (gauge symmetries) [9] on a level where the gauge fields are treated beyond the classical field level, i.e., on the propagator level.

It can be shown though, that on top of any solution of a $\Phi$-derivable approximation which is constructed from a symmetric Lagrangian there exists a non-perturbative effective action $\Gamma_{\text {eff }}[\varphi]$ which generates proper vertex functions in the same sense as the 1PI effective action. These $e x-$ ternal vertex functions fulfill the Ward-Takahashi identities of the underlying symmetry. However, in general they coincide with the self-consistent ones only up to one-point order. This fact especially ensures that the expectation values of Noether currents are conserved for the $\Phi$-derivable approximations. Thus usually the so generated external self-energy and higher vertex functions

are different from the $\Phi$-derivable expressions. Details on these considerations will be given in a forthcoming paper []].

\section{Acknowledgments}

We are grateful to J. Berges, J. P. Blaizot, G. E. Brown, P. Danielewicz, B. Friman, Yu. Ivanov, M. Lutz, M. A. Nowak and D. Voskresensky for fruitful discussions and suggestions at various stages of this work.

[1] H. van Hees and J. Knoll, Phys. Rev. D65, 025010 (2002), URL http://arXiv.org/abs/hep-ph/0107200.

[2] G. Baym, Phys. Rev. 127, 1391 (1962), URL http://prola.aps.org/abstract/PR/v127/i4/p1391_1.

[3] J. Luttinger and J. Ward, Phys. Rev. 118, 1417 (1960), URL http://prola.aps.org/abstract/PR/v118/i5/p1417__.

[4] M. Cornwall, R. Jackiw, and E. Tomboulis, Phys. Rev. D10, 2428 (1974), URL http://prola.aps.org/abstract/PRD/v10/i8/p2428_1.

[5] E. Braaten and E. Petitgirard (2001), http://arXiv.org/abs/hep-ph/0107118.

[6] G. Baym and G. Grinstein, Phys. Rev. D15, 2897 (1977), URL http://prola.aps.org/abstract/PRD/v15/i10/p2897_1.

[7] H. van Hees and J. Knoll, Phys. Rev. D (2001), in preparation.

[8] A. Peshier, Phys. Rev. D63, 105004 (2001), URL http://arXiv.org/abs/hep-ph/0011250. 
[9] H. van Hees and J. Knoll, Nucl. Phys. A683, 369 (2001), URL http://arXiv.org/abs/hep-ph/0007070.

[10] J. Schwinger, J. Math. Phys 2, 407 (1961).

[11] L. Keldysh, ZhETF 47, 1515 (1964), [Sov. Phys JETP 201965 1018].

[12] Y. B. Ivanov, J. Knoll, and D. N. Voskresensky, Nucl. Phys. A657, 413 (1999), URL http://arXiv.org/abs/hep-ph/9807351.

[13] G. 't Hooft, Nucl. Phys. B61, 455 (1973).

[14] W. A. Bardeen, A. J. Buras, D. W. Duke, and T. Muta, Phys. Rev. D18, 3998 (1978), URL http://prola.aps.org/abstract/PRD/v18/i11/p3998_1.

[15] N. N. Bogoliubov and O. S. Parasiuk, Acta Math. 97, 227 (1957).

[16] W. Zimmermann, Commun. Math. Phys. 15, 208 (1969).

[17] P. Ramond, Field Theory: A Modern Primer (Addison-Wesley Publishing Company, Inc., 1989), 2nd ed.

[18] Y. B. Ivanov, J. Knoll, and D. N. Voskresensky, Nucl. Phys. A672, 313 (2000), URL http://arXiv.org/abs/nucl-th/9905028.

[19] J. Berges (2001), URL http://de.arXiv.org/abs/hep-ph/0105311.

[20] J. Berges and J. Cox, Phys. Lett. B517, 369 (2001), URL http://de.arXiv.org/abs/hep-ph/0006160.

[21] G. Aarts and J. Berges, Phys. Rev. D64, 105010 (2001), URL http://de.arXiv.org/abs/hep-ph/0103049. 